\title{
SAID analysis of meson photoproduction: Determination of neutron and proton EM couplings
}

\author{
Igor Strakovsky $^{1, a}$, William Briscoe ${ }^{1}$, Alexander Kudryavtsev ${ }^{2,1}$, Vladimir Tarasov ${ }^{2}$ \\ and Ron Workman ${ }^{1}$ \\ ${ }^{1}$ Institute for Nuclear Studies, Department of Physics, The George Washington University, Washington, \\ DC 20052, USA \\ ${ }^{2}$ Institute of Theoretical and Experimental Physics, Moscow 117259, Russia
}

\begin{abstract}
We present an overview of the GW SAID group effort to analyze on new pion photoproduction on both proton- and neutron-targets. The main database contribution came from the recent CLAS and MAMI unpolarized and polarized measurements. The differential cross section for the processes $\gamma n \rightarrow \pi^{-} p$ was extracted from new measurements accounting for Fermi motion effects in the impulse approximation (IA) as well as $\mathrm{NN}$ - and $\pi \mathrm{N}$ effects beyond the IA. The electromagnetic coupling results are compared to other recent studies.
\end{abstract}

\section{SAID for baryon spectroscopy}

The properties of the resonances for the non-strange sector have been determined almost entirely from the results of $\pi \mathrm{N}$ elastic scattering analyses [1]. Meson photoproduction reactions have mainly served to fix electromagnetic (EM) couplings. With the refinement of multichannel fits and the availability of highprecision photoproduction data for both single- and double-meson production, identifications of some new states have emerged mainly due to evidence from reactions not involving single-pion-nucleon initial or final states [1]. The GW SAID $N^{*}$ program consists of $\pi N \rightarrow \pi N, \gamma N \rightarrow \pi N$, and $\gamma^{*} p \rightarrow \pi N$ components as was established by Dick Arndt on 1997. Assuming dominance of two hadronic channels [ $\pi \mathrm{N}$ elastic and $\pi N \rightarrow \eta N$ ], we parametrize $\gamma^{*} p \rightarrow \pi N$ in terms of $\pi N \rightarrow \pi N$ amplitudes ([2] and references therein). Most of the pion photoproduction analyses use SAID $\pi \mathrm{N}$ partial-wave analysis (PWA) outcome [3] or its modification as input for the constraint as well. However, beyond $\pi \mathrm{N}$ elastic scattering, single-pion photoproduction remains the most studied source of resonance information. Much of the effort aimed at providing complete or nearly complete information for meson-nucleon photoproduction reactions has been directed to measuring double-polarization observables. However, often overlooked is that the data coverage for several single-polarization observables, also vital in determining the properties of the nucleon resonance spectrum, still remains incomplete.

\footnotetext{
ae-mail: igor@gwu.edu
}

This is an Open Access article distributed under the terms of the Creative Commons Attribution License 4.0, which permits unrestricted use, distribution, and reproduction in any medium, provided the original work is properly cited. 

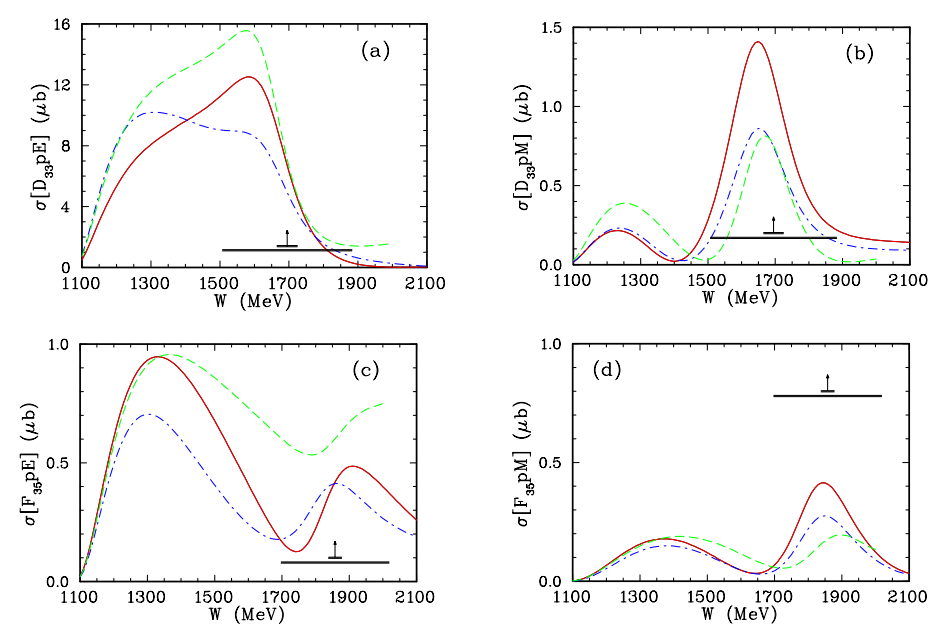

Figure 1. Partial cross sections for multipoles with the largest change was found after including the new CLAS data in the fit, $\Delta(1700) 3 / 2^{-}$and $\Delta(1905) 5 / 2^{+}$. Solid (dash-dotted) lines correspond to the SAID DU13 [8] (CM12 [2]) solution. Dashed lines give MAID07 [9], Vertical arrows indicate resonance energies $\mathrm{W}_{R}$ and horizontal bars show full $\Gamma_{\pi N}$ widths associated with the SAID $\pi$ N solution WI08 [3].

Here we focus on the single-pion production data and note that a complete solution requires couplings from both charged and neutral resonances $[4,5]$, the latter requiring $\pi^{-} p$ and $\pi^{0} n$ photoproduction off a neutron target, typically a neutron bound in a deuteron target. Extraction of the two-body $\left(\gamma n \rightarrow \pi^{-} p\right.$ and $\gamma n \rightarrow \pi^{0} n$ ) cross sections requires the use of a model-dependent nuclear correction, which mainly comes from final-state interactions (FSI) [6]. As a result, our knowledge of the neutral resonance couplings is less precise than that of the charged values for well-known lowlaying baryons. The uncertainties for such kind of neutral states with $\mathrm{J}^{P}=\frac{1}{2}$, for instance, $\mathrm{N}(1440) 1 / 2^{+}$, $\mathrm{N}(1535) 1 / 2^{-}$, and $\mathrm{N}(1650) 1 / 2^{-}$vary from $25 \%$ to $140 \%$ [1]. Some of the $\mathrm{N}^{*}$ baryons $\left[\mathrm{N}(1675) 5 / 2^{-}\right.$, for instance] have stronger EM couplings to the neutron than to the proton, but parameters are very uncertain $\left(N^{*} \rightarrow \gamma p:+0.019 \pm 0.008 \mathrm{GeV}^{-1 / 2}\right.$ while $\left.N^{*} \rightarrow \gamma n:-0.043 \pm 0.012 \mathrm{GeV}^{-1 / 2}[1]\right)$. Then, PDG12 estimates for the $A_{1 / 2}$ and $A_{3 / 2}$ proton decay amplitudes of the $\mathrm{N}(1720) 3 / 2^{+}$state are consistent with zero, while the recent SAID determination [2] gives small but non-vanishing values. Other unresolved issues relate to the second $\mathrm{P}_{11}, \mathrm{~N}(1710) 1 / 2^{+}$, that we do not seen in the recent SAID $\pi \mathrm{N}$ PWA [3] contrary to the findings of other PWAs referenced by PDG12 [1].

\section{Pion photoproduction off the proton}

The overall SAID $\chi^{2}$ has remained stable $\left(\chi^{2} /\right.$ data $\left.=2.1\right)$ against the growing database, which has increased by a factor of 2 since 1995 (13.4 $\mathrm{k}$ up to $27.3 \mathrm{k}$ data points) [7]. Most of this increase coming from photon-tagging facilities. More complete data sets for double- and single-polarization observables for pion photoproduction can offer important constraints on analyses of the photoproduction reaction.

Using linearly polarized photons and an unpolarized target, CLAS provides a large set of beam asymmetry $\Sigma$ measurements for $\gamma p \rightarrow \pi^{0} p$ and $\gamma p \rightarrow \pi^{+} n$ from $\mathrm{E}_{\gamma}=1.100$ and up to $1.860 \mathrm{GeV}$ in laboratory photon energy, corresponding to a $\mathrm{CM}$ energy $\mathrm{W}$ range of $1.7-2.1 \mathrm{GeV}\left(\theta=30-150^{\circ}\right.$ of pion production angle in $\mathrm{CM}$ ) [8]. Its contribution to the world database is more than doubled [7]. In Fig. 1, we show the effect of new CLAS $\Sigma$ measurements in terms of partial cross sections from SAID (CM12 [2] and recent DU13 [8] included new CLAS data) and MAID [9]. While the CM12 and DU13 solutions differ over the energy range of the recent CLAS experiment, the resonance couplings are fairly 
Table 1. Proton helicity amplitudess $p A_{1 / 2}$ and $p A_{3 / 2}$ (in $\left[(\mathrm{GeV})^{-1 / 2} \times 10^{-3}\right]$ units).

\begin{tabular}{|c|c|c|c|}
\hline Resonance & $p A_{1 / 2}$ & $p A_{3 / 2}$ & Ref. \\
\hline$\Delta(1700) 3 / 2^{-}$ & $132 \pm 5$ & $108 \pm 5$ & SAID DU13 [8] \\
& $105 \pm 5$ & $92 \pm 4$ & SAID CM12 [2] \\
& $160 \pm 20$ & $165 \pm 25$ & BnGa12 [10] \\
& $58 \pm 10$ & $97 \pm 8$ & Kent12 [11] \\
& 226 & 210 & MAID [9] \\
& $104 \pm 15$ & $85 \pm 22$ & PDG12 [1] \\
\hline$\Delta(1905) 5 / 2^{+}$ & $20 \pm 2$ & $-49 \pm 5$ & SAID DU13 [8] \\
& $19 \pm 2$ & $-38 \pm 4$ & SAID CM12 [2] \\
& $25 \pm 5$ & $-49 \pm 4$ & BnGa12 [10] \\
& $66 \pm 18$ & $-223 \pm 29$ & Kent12 [11] \\
& 18 & -28 & MAID [9] \\
& $26 \pm 11$ & $-45 \pm 20$ & PDG12 [1] \\
\hline
\end{tabular}

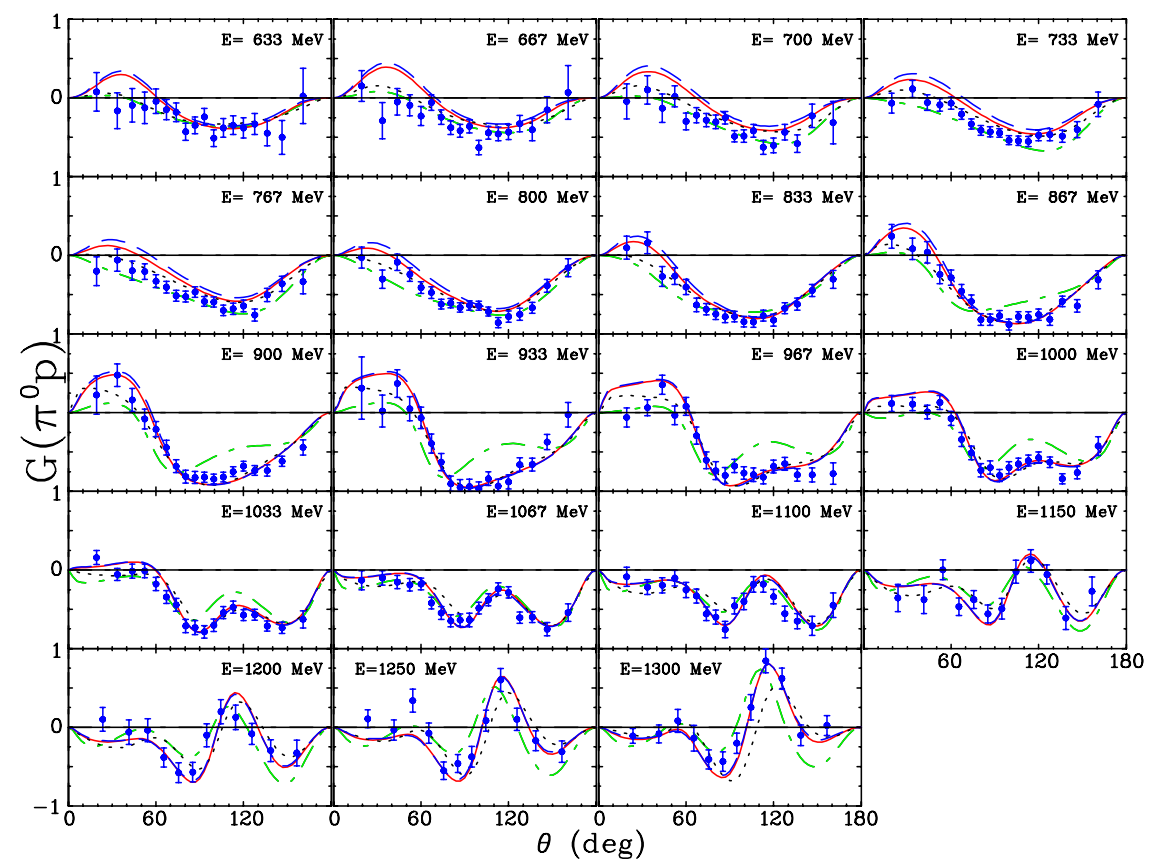

Figure 2. The double-polarization observable $G$ as a function of pion production angle in CM. Dashed (solid) lines correspond to the SAID (DU13 [8] and preliminary solution included new CB-ELSA $G$ measuremenents. Dotted (dash-dotted) lines give BnGa12 [10] (MAID07 [9]).

stable. The largest change is found for the $\Delta(1700) 3 / 2^{-}$and $\Delta(1905) 5 / 2^{+}$states, for which the various analyses disagree significantly in terms of photo-decay amplitudes (Table 1).

With the inclusion of new high-precision data, our fits are becoming more stable and predictive. Plots of recent double polarized $G$ data, covered $\mathrm{E}_{\gamma}=630-1300 \mathrm{MeV}$ and $\theta=20-160^{\circ}$, in Fig. 2 from CB-ELSA [12] show that the SAID CM12 fit gives a good prediction of this quantity. We have recently analyzed $C_{x^{\prime}}\left(\mathrm{E}_{\gamma}=460-1340 \mathrm{MeV}\right.$ and $\left.\theta=75-140^{\circ}\right)$ [13] and preliminary $F$ and $T$ data $\left(\mathrm{E}_{\gamma}=440-\right.$ $1430 \mathrm{MeV}$ and $\theta=30-160^{\circ}$ ) [14] from Mainz, finding a similarly quantitative level of agreement. 


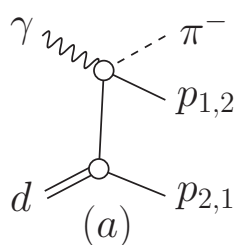

(a)

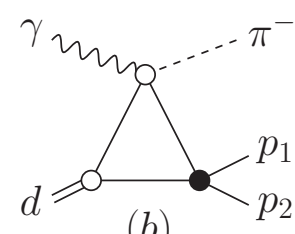

(b)

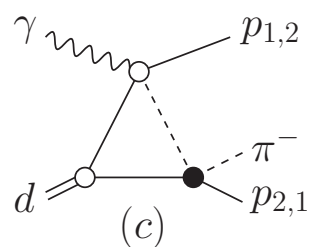

Figure 3. Feynman diagrams for the leading terms of the $\gamma d \rightarrow \pi^{-} p p$ amplitude. (a) IA, (b) $p p$-FSI, and (c) $\pi N-$ FSI. Filled black circles show FSI vertices. Wavy, dashed, solid, and double lines correspond to the photons, pions, nucleons, and deuterons, respectively.

\section{Pion photoproduction off the neutron}

In addition to being less precise, experimental data for neutron-target photoreactions are much less abundant than those utilizing a proton target, constituting only about $15 \%$ of the present SAID database [7]. At low to intermediate energies, this lack of neutron-target data is partially compensated by experiments using pionic beams, e.g., $\pi^{-} p \rightarrow \gamma n$, as has been measured, for example, by the Crystal Ball Collaboration at BNL [15] for the inverse photon energy $\mathrm{E}_{\gamma}=285-690 \mathrm{MeV}$ and $\theta=40-150^{\circ}$, where $\theta$ is the inverse production angle of pion in the $\mathrm{CM}$ frame. This process is free from complications associated with the deuteron target. However, the disadvantage of using the reaction $\pi^{-} p \rightarrow \gamma n$ for the pion photoproduction study is the 5 to 500 times larger cross sections for $\pi^{-} p \rightarrow \pi^{0} n \rightarrow \gamma \gamma n$, depending on $\mathrm{E}_{\gamma}$ and $\theta$.

We extract the $\gamma n \rightarrow \pi^{-} p$ cross section on free nucleon from the deuteron data in the quasi-free (QF) kinematic region of the $\gamma d \rightarrow \pi^{-} p p$ reaction with fast knocked-out proton and slow proton-spectator assumed not to be involved in the pion production process. In this, so-called impulse approximation (IA) [16], the reaction mechanism corresponds to the diagram in Fig. 3a. There are 2 critical factors to be taken into account when using this approach: (i) the neutron is bound and (ii) there are NN- and $\pi N$-FSI effects.

Item (i) means that the effective mass of the neutron is not equal to the mass of the free neutron. In our former analyses $[17,18]$, the $\gamma n \rightarrow \pi^{-} p$ amplitude for a given $\mathrm{E}_{\gamma}$ and CM pion production angle $\theta$ is assumed to be the same as on a free neutron at rest. That is why the cross section obtained should be considered as an average over energies around $\mathrm{E}_{\gamma}$. The size of the averaging region is determined by a smearing of the energy owing to the Fermi-motion in the deuteron. The typical scale here is $20 \mathrm{MeV}$ in energy.

Item (ii) corresponds to the inclusion of the FSI corrections. Their leading terms correspond to Feynman diagrams shown on Fig. 3(b,c). Determinations of the $\gamma d \rightarrow \pi^{-} p p$ differential cross section, with the FSI taken into account (all the diagrams on Fig. 3, were included) were done recently for the CLAS [17] and MAMI-B [18] $\gamma d \rightarrow \pi^{-} p p$ data. The SAID phenomenological amplitudes for $\gamma N \rightarrow \pi N$ [19], NN-elastic [20], and $\pi N$-elastic [3] were used as inputs to calculate the diagrams in Fig. 3. The Bonn potential [21] was used for the deuteron description.

Recently, we applied our FSI corrections [22] to CLAS $\gamma d \rightarrow \pi^{-} p p$ data $\left(\mathrm{E}_{\gamma}=1050-2700 \mathrm{MeV}\right.$ and $\theta=30-160^{\circ}$ ) [23] to get elementary cross sections for $\gamma n \rightarrow \pi^{-} p$ [17]. New CLAS differential cross sections are quadrupling the world database for $\gamma n \rightarrow \pi^{-} p$ above $1 \mathrm{GeV}$. The FSI correction factor for the CLAS kinematics was found to be small, $\Delta \sigma / \sigma<10 \%$. However, these new cross sections departed significantly from our predictions at the higher energies, and greatly modified the fit result, which allows to determine new neutron couplings (Table 2).

In our recent study [18], we addressed to the differential cross section measurements for $\gamma n \rightarrow \pi^{-} p$ in the $\Delta$-isobar region. The data came from MAMI-B $\left(\mathrm{E}_{\gamma}=300-455 \mathrm{MeV}\right.$ and $\left.\theta=60-140^{\circ}\right)$ [24]. At energies dominated by the $\Delta$-resonance, the isospin $\mathrm{I}=3 / 2$ multipoles are constrained by extensive 
Table 2. Neutron helicity amplitudes $n A_{1 / 2}$ and $n A_{3 / 2}$ (in $\left[(\mathrm{GeV})^{-1 / 2} \times 10^{-3}\right]$ units).

\begin{tabular}{|cc|ccc|c|}
\hline Resonance & $n A_{1 / 2}$ & Resonance & $n A_{1 / 2}$ & $n A_{3 / 2}$ & Ref. \\
\hline$N(1535) 1 / 2^{-}$ & $-58 \pm 6$ & $N(1520) 3 / 2^{-}$ & $-46 \pm 6$ & $-115 \pm 5$ & SAID GB12 [17] \\
& $-60 \pm 3$ & & $-47 \pm 2$ & $-125 \pm 2$ & SAID SN11 [25] \\
& $-93 \pm 11$ & & $-49 \pm 8$ & $-113 \pm 12$ & BnGa13 [26] \\
& $-49 \pm 3$ & & $-38 \pm 3$ & $-101 \pm 4$ & Kent12 [11] \\
$N(1650) 1 / 2^{-}$ & $-46 \pm 27$ & & $-59 \pm 9$ & $-139 \pm 11$ & PDG12 [1] \\
& $-40 \pm 10$ & $N(1675) 5 / 2^{-}$ & $-58 \pm 2$ & $-80 \pm 5$ & SAID GB12 [17] \\
& $25 \pm 20$ & & $-42 \pm 2$ & $-60 \pm 2$ & SAID SN11 [25] \\
& & $-60 \pm 7$ & $-88 \pm 10$ & BnGa13 [26] \\
$N(1440) 1 / 2^{+}$ & $-15 \pm 21$ & & $-40 \pm 4$ & $-68 \pm 4$ & Kent12 [11] \\
& $48 \pm 4$ & $N(1680) 5 / 2^{+}$ & $26 \pm 4$ & $-29 \pm 2$ & SAID GB12 [17] \\
& & $50 \pm 4$ & $-47 \pm 2$ & SAID SN11 [25] \\
$43 \pm 12$ & & $34 \pm 6$ & $-44 \pm 9$ & BnGa13 [26] \\
$40 \pm 5$ & & $29 \pm 2$ & $-59 \pm 2$ & Kent12 [11] \\
$40 \pm 10$ & & $29 \pm 10$ & $-33 \pm 9$ & PDG12 [1] \\
\hline
\end{tabular}

studies performed using proton targets. The forward peaking structure is due largely to the Born contribution, which is well known. As a result, one would expect models to give predictions within a tight range.

We have included the new neutron cross sections from the CLAS and MAMI-B experiments in a number of multipole analyses covering incident photon energies up to $2.7 \mathrm{GeV}$, using the full SAID database [7], in order to gauge the influence of these measurements, as well as their compatibility with previous experiments. The solution, GB12 [17], uses the same fitting form as our recent SN11 solution [25]. A second fit, GZ12, instead used the recently proposed form based on a unified ChewMandelstam parametrization of the GW DAC fits to both $\pi \mathrm{N}$ elastic scattering and photoproduction [2].

Table 2 shows that the new SAID GB12 $n A_{1 / 2}$ and $n A_{3 / 2}$ helicities sometimes have a significant deviation from the previous SAID SN11 [25] determination and PDG12 [1] values, e.g., for $N(1650) 1 / 2^{-}, N(1675) 5 / 2^{-}$, and $N(1680) 5 / 2^{+}$. While BnGa13 group [26] used the same (almost) data to fit them as we are while BnGa13 has several new ad hoc resonances. Meanwhile, BnGa13 determination is different for $N(1535) 1 / 2^{-}, N(1650) 1 / 2^{-}$, and $N(1680) 5 / 2^{+}$.

\section{Summary}

Future progress in the database development is expecting from tagged-photon fasilities as JLab, MAMIC, SPring-8, CB-ELSA, and ELPH. Partial-wave analyses will clearly benefit from the constraints provided by these new data, which highlight the importance of new polarization observables in providing a stringent test of PWA, even in kinematic regions where a large number of cross section and polarization observables are already present in the world database. An accurate PWA must ultimately describe a complete set of observables. The current data and future experiments exploiting these polarimetry developments at large acceptance detectors will be a key part to achieving this complete measurement.

In this regard, future experiments to measure unpolarized and the spin polarization of neutrons are already planned at MAMI-C. Measurements of such observables with large acceptance are crucial to the world program aiming to determine the excitation spectrum of the nucleon.

We proposed to perform a precision measurement of $d \sigma / d \Omega$ in the reactions $\gamma d \rightarrow \pi^{-} p p$ and $\gamma d \rightarrow \pi^{0} n p$ in the tagged-photon energy region from threshold to $800 \mathrm{MeV}$ [27] and then to $1500 \mathrm{MeV}$ [28]. The $d \sigma / d \Omega$ for the processes $\gamma p \rightarrow \pi^{-} p$ and $\gamma p \rightarrow \pi^{0} n$ will be extracted from these CB@ MAMI$\mathrm{C}$ measurements accounting for Fermi motion effects in IA [16] as well as NN- and $\pi$ N-FSI effects beyond the IA. Data below $800 \mathrm{MeV}$ were taken in March of 2013 and analysis is in progress. 
Consequential calculations of the FSI corrections, as developed by our GW-ITEP Collaboration, will be applied. We will extend our FSI code [22] to extract $\gamma n \rightarrow \pi^{0} n$ data from $\gamma d \rightarrow \pi^{0} n p$ measurements as well. Polarized measurements will help to bring more physics in. FSI corrections need to apply.

This work was supported in part by the U. S. DOE Grant No. DE-FG02-99ER41110.

\section{References}

[1] J. Beringer et al. (Particle Data Group), Phys. Rev. D 86, 010001 (2012)

[2] R. Workman et al., Phys. Rev. C 86, 015202 (2012)

[3] R.A. Arndt et al., Phys. Rev. C 74, 045205 (2006)

[4] K.M. Watson, Phys. Rev. 95, 228 (1954)

[5] R.L. Walker, Phys. Rev. 182, 1729 (1969)

[6] A.B. Migdal, JETP 1, 2 (1955); K.M. Watson, Phys. Rev. 88, 1163 (1952)

[7] Institute of Nuclear Studies of GW Database (W.J. Briscoe, I.I. Strakovsky, and R.L. Workman); http://gwdac.phys.gwu.edu/analysis/pr_analysis.html

[8] M. Dugger et al. (CLAS Collaboration), Phys. Rev. C 88, 065203 (2013)

[9] D. Drechsel et al., Eur. Phys. J. A 34, 69 (2007)

[10] A. Anisovich et al., Eur. Phys. J. A 48, 15 (2012)

[11] M. Shrestha and D.M. Manley, Phys. Rev. C 86, 055203 (2012)

[12] A. Thiel (CBELSA/TAPS Collaboration) et al., Phys. Rev. Lett. 109, 102001 (2012); R.L. Workman et al., Phys. Rev. Lett. 110, 169101 (2013); A. Thiel (CBELSA/TAPS Collaboration) et al., Phys. Rev. Lett. 110, 169102 (2013)

[13] M.H. Sikora (A2 Collaboration) et al., arXiv:1309.7898

[14] V. Kashevarov, Proceedings of MENU 2013 workshop

[15] A. Shafi et al. (Crystal Ball Collaboration), Phys. Rev. C 70, 035204 (2004)

[16] G. Chew and M. Goldberger, Phys. Rev. 87, 778 (1952)

[17] W. Chen et al., Phys. Rev. C 86, 015206 (2012)

[18] W.J. Briscoe et al., Phys. Rev. C 86, 065207 (2012)

[19] M. Dugger et al. (CLAS Collaboration), Phys. Rev. C 76, 025211 (2007)

[20] R.A. Arndt et al., Phys. Rev. C 76, 025211 (2007)

[21] R. Machleidt, K. Holinde, and C. Elster, Phys. Rept. 140, 1 (1987)

[22] V.E. Tarasov et al., Phys. Rev. C 84, 035203 (2011)

[23] W. Chen et al. (CLAS Collaboration), Phys. Rev. Lett. 103, 012301 (2009)

[24] J. Ahrens, et al. (GDH and A2 Collaborations), Eur. Phys. J. A 44, 189 (2010)

[25] R. Workman et al., Phys. Rev. C 85, 025201 (2012)

[26] A. Anisovich et al., Eur. Phys. J. A 49, 67 (2013)

[27] Meson production off the deuteron, Spokespersons: W.J. Briscoe and I.I. Strakovsky, MAMI Proposal MAMI-A2-02/12, Mainz, Germany, 2012

[28] Meson production off the deuteron. II, Spokespersons: W.J. Briscoe, V.V. Kulikov, K. Livingston, and I.I. Strakovsky, MAMI Proposal MAMI-A2-02/13, Mainz, Germany, 2013 